\title{
The effectiveness of interventions to treat obesity in survivors of childhood brain tumors: a systematic review protocol
}

\author{
Kuan-Wen Wang ${ }^{1,2}$, Marlie Valencia ${ }^{1,2}$, Laura Banfield ${ }^{3}$, Ruth Chau ${ }^{1,2}$, Adam Fleming ${ }^{1,4}$, Sheila K. Singh ${ }^{5,6}$, \\ Sarah Burrow ${ }^{7}$, Russell J. de Souza ${ }^{8}$, Lehana Thabane ${ }^{1,8,9,10,11}$ and M. Constantine Samaan ${ }^{1,2,8^{*}}$
}

\begin{abstract}
Background: Pediatric brain tumors are the most common solid tumors in children. Advances in understanding the hallmarks of cancer biology and novel therapies have led to an increasing number of survivors of childhood brain tumors (SCBT). However, these survivors are at an increased risk of obesity and cardiometabolic disorders that affect their quality of life and lifespan. It is important to define effective strategies to treat and prevent obesity in this population. This systematic review aims to investigate the effectiveness of lifestyle interventions, pharmacotherapy, and bariatric surgery on treating obesity in SCBT.

Methods: Searches will be conducted in PubMed, MEDLINE, EMBASE, PsycINFO, SPORTDiscus, CINAHL, Cochrane Database of Systematic Review, Cochrane Central Register of Controlled Trials (CENTRAL), and Database of Abstracts of Reviews of Effect (DARE). In addition, ClinicalTrials.gov and ProQuest Dissertations and Theses A\&I will be searched to identify relevant gray literature. The reference lists of eligible articles will be searched for additional studies. All screening, quality assessment, and data abstraction will be done independently by two reviewers. We will perform meta-analysis if there are sufficient studies.

Discussion: This review will summarize evidence for the effectiveness of interventions used to reduce obesity risk in SCBT. This has significant implications for SCBT, as it can identify gaps in knowledge and provide insights into the development of new interventions to manage obesity in survivors, which may improve their outcomes.
\end{abstract}

Systematic review registration: PROSPERO CRD42015025909

Keywords: Systematic review, Protocol, Obesity, Intervention, Children, Brain tumor, Brain tumor survivors, Cancer survivorship

\section{Introduction}

Brain tumors are the most common solid tumors in children and constitute up to $20 \%$ of childhood cancers [1]. Significant breakthroughs in understanding the hallmarks of cancer biology, coupled with advances in diagnostic imaging and improved therapies, have enhanced the survival rates of these children $[2,3]$.

As the number of survivors of childhood brain tumors (SCBT) increased, it has become apparent that survivors

\footnotetext{
*Correspondence: samaanc@mcmaster.ca

'Department of Pediatrics, McMaster University, 1280 Main Street West, HSC-3A57, Hamilton, Ontario L8S 4K1, Canada

2Division of Pediatric Endocrinology, McMaster Children's Hospital, 1280 Main

Street West, HSC-3A57, Hamilton, Ontario L8S 4K1, Canada

Full list of author information is available at the end of the article
}

remain at risk of premature mortality $[4-6]$ and the development of multiple comorbidities [7, 8]. Many SCBT develop chronic health conditions within years of their initial diagnosis [9], and one such morbidity is obesity [10-13]. In one study, obesity was reported in $36.5 \%$ of SCBT, compared to $29 \%$ in the general population [14, 15]. In the general population, the annual healthcare expenditures of obese individuals are about US $\$ 1360$ higher than for their non-obese counterparts [16], and this is likely to be replicated in SCBT.

Addressing obesity in SCBT is crucial, as it increases the risk of cardiometabolic disorders in a similar fashion to the general population, and may contribute to premature mortality $[17,18]$. Obesity is an independent risk 
factor for decreased survival in some children with brain tumors [19]. Understanding the drivers of obesity in SCBT will allow the development of precision-based strategies for reducing the risk of obesity and its cardiometabolic comorbidities, which in turn may improve the quality of life and lifespan of SCBT.

Obesity in SCBT is multifactorial and can be related to altered energy intake [20,21], reduced mobility and physical activity [22-25], hypothalamic-pituitary damage [11], pituitary hormone deficiencies [26], sleep problems [27], vision problems, imbalance and pain [8, 28], mental health issues, and medications, e.g., antidepressants [29].

As obese children are likely to become obese adults [30-34], it is important to develop effective interventions to manage obesity from an early age. The purpose of this systematic review is to evaluate current evidence of effectiveness of interventions to manage obesity in SCBT.

\section{Research question}

In survivors of childhood brain tumors, are the current interventions including lifestyle intervention, pharmacotherapy, and bariatric surgery effective in managing obesity?

\section{Study objectives}

1) Measure the effectiveness of lifestyle interventions, pharmacotherapy, and bariatric surgery in the treatment of obesity in SCBT

2) Conduct a meta-analysis of primary studies, if appropriate, to gain a more precise estimate of the effectiveness of different strategies in managing obesity

3) Critically appraise existing evidence and identify gaps in the literature to provide future research directions

\section{Methods}

The protocol for this systematic review is developed and reported with guidance from the Preferred Reporting Items for Systematic Review and Meta-Analysis-Protocols (PRISMA-P) statement (Additional file 1) [35].

\section{Eligibility criteria}

This review will include studies involving boys and girls who are overweight or obese (BMI z-score $\geq 85$ th percentile) [36], with a diagnosis of brain tumor made under the age of 18 years. Randomized controlled trials (RCTs), quasi-RCTs, prospective or retrospective cohort studies, case-control studies, cross-sectional studies, and controlled or uncontrolled studies with before-and-after comparisons will be included [37].
There will be no restriction to the language or timing of publication. Conference proceedings, congress reports, and editorials will be hand searched for suggested relevant studies. We will exclude interim analyses, case reports, and pilot studies.

In studies where SCBT are included in an intervention with other cancer types, we will extract data for the brain tumor subgroup. If the data from subgroups are not published or pooled with data from survivors of other cancers, we will attempt to contact the authors to obtain the subgroup data.

The interventions included in the study are

- Lifestyle intervention: any form of modifications in subjects' daily life including their dietary patterns, physical activity, and eating behaviors

- Pharmacotherapy: any administration of medications

- Bariatric surgery: any surgical approach performed with the intention of treating obesity, including adjustable gastric banding, sleeve gastrectomy, biliopancreatic diversion with duodenal switch, and gastric bypass

Studies that are entered into the databases up to February 1, 2016, will be screened for eligibility. The search will be updated to capture recently published literature.

\section{Outcome measures Primary outcome}

The primary outcome in this review is BMI z-score change from baseline to the end of the intervention and/ or at follow-up.

\section{Secondary outcomes}

Secondary outcomes include changes in waist and hip circumference, waist-to-hip ratio, waist-to-height ratio, body fat percentage, and blood pressure as reported. We will also report changes in diabetes status, insulin resistance, and non-alcoholic fatty liver disease, if available. In addition, we will document changes in lipid levels including high-density lipoprotein, low-density lipoprotein, cholesterol, and triglycerides, if reported.

We will also abstract any adverse events observed during the study. Adverse events directly related to lifestyle interventions include back and shoulder pain, musculoskeletal injuries, and others [38, 39]. Adverse events for the pharmacological agents include insomnia, headaches, hypertension, and others [40]. Adverse outcomes for bariatric surgery include surgical complications, perioperative outcomes, and mortality as defined previously [41]. Additional adverse events will be included as reported. 
Table 1 Search strategy for MEDLINE

\begin{tabular}{|c|c|}
\hline 1 & $\exp$ Child/ \\
\hline 2 & child*.mp. \\
\hline 3 & p?ediatric*.mp. \\
\hline 4 & exp Adolescent/ \\
\hline 5 & adolescen*.mp. \\
\hline 6 & youth*.mp. \\
\hline 7 & exp Adult/ \\
\hline 8 & adult*.mp. \\
\hline 9 & Young Adult/ \\
\hline 10 & 1 or 2 or 3 or 4 or 5 or 6 or 7 or 8 or 9 \\
\hline 11 & exp Brain Neoplasms/ \\
\hline 12 & exp Cranial Nerve Neoplasms/ \\
\hline 13 & exp Neuroectodermal Tumors/ \\
\hline 14 & cerebroma*.mp. \\
\hline 15 & exp Glioma/ \\
\hline 16 & glioma*.mp. \\
\hline 17 & astrocytoma*.mp. \\
\hline 18 & oligoastrocytoma*.mp. \\
\hline 19 & astroglioma*.mp. \\
\hline 20 & glioblastoma*.mp. \\
\hline 21 & retinoblastoma*.mp. \\
\hline 22 & pinealoma*.mp. \\
\hline 23 & pineoblastoma*.mp. \\
\hline 24 & pinealoblastoma*.mp. \\
\hline 25 & pinealblastoma*.mp. \\
\hline 26 & pineal blastoma*.mp. \\
\hline 27 & pineocytoma*.mp. \\
\hline 28 & pinealocytoma*.mp. \\
\hline 29 & craniopharyngioma*.mp. \\
\hline 30 & ependymoma*.mp. \\
\hline 31 & subependymoma*.mp. \\
\hline 32 & ependymoblastoma*.mp. \\
\hline 33 & ganglioglioma*.mp. \\
\hline 34 & gliosarcoma*.mp. \\
\hline 35 & medulloblastoma*.mp. \\
\hline 36 & exp Germinoma/ \\
\hline 37 & germinoma*.mp. \\
\hline 38 & Meningioma/ \\
\hline 39 & meningioma*.mp. \\
\hline 40 & oligodendroglioma*.mp. \\
\hline 41 & exp Neurofibromatoses/ \\
\hline 42 & neurofibromatos*.mp. \\
\hline 43 & $\mathrm{PNET}^{*} \cdot \mathrm{mp}$ \\
\hline 44 & neurocytoma*.mp. \\
\hline 45 & choroid plexus papilloma*.mp. \\
\hline
\end{tabular}

Table 1 Search strategy for MEDLINE (Continued)

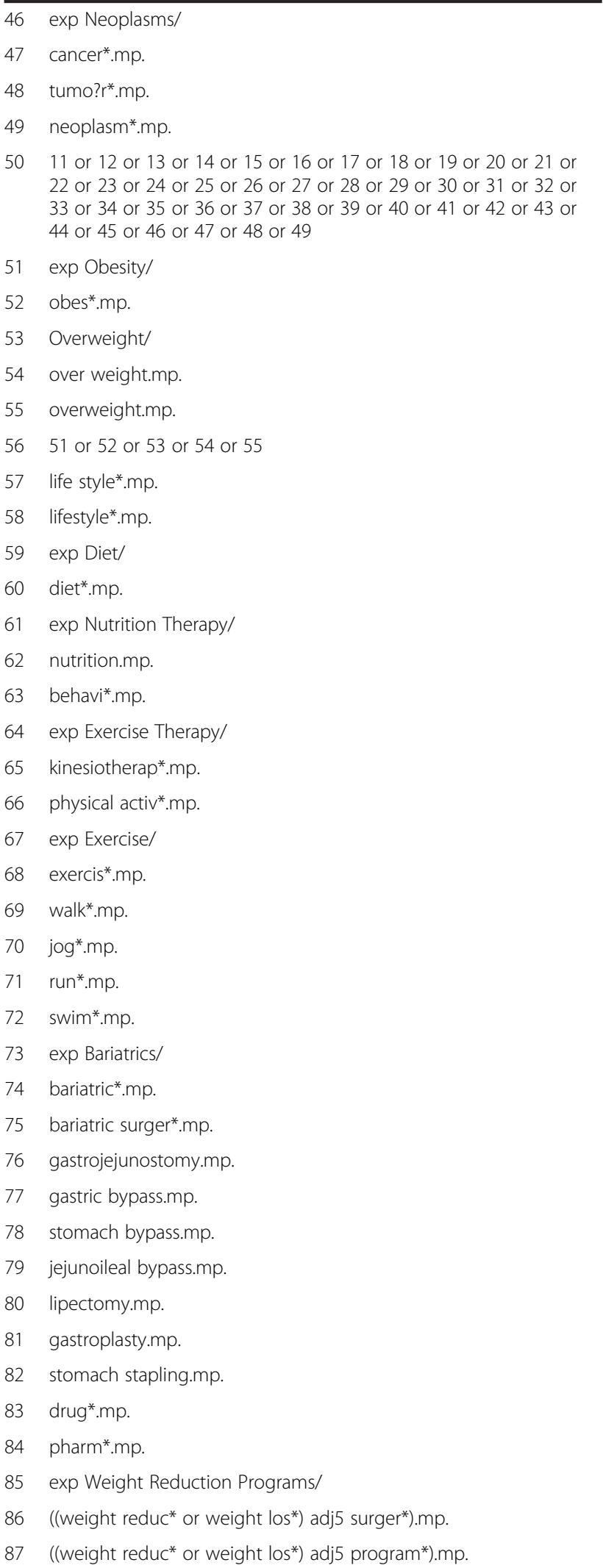


Table 1 Search strategy for MEDLINE (Continued)

\begin{tabular}{l}
88 or 58 or 59 or 60 or 61 or 62 or 63 or 64 or 65 or 66 or 67 or \\
68 or 69 or 70 or 71 or 72 or 73 or 74 or 75 or 76 or 77 or 78 or \\
79 or 80 or 81 or 82 or 83 or 84 or 85 or 86 or 87 \\
89 Body Weight/ \\
90 body mass*.mp. \\
91 BMl.mp. \\
92 exp Body Weight Changes/ \\
93 exp Body Weights and Measures/ \\
94 body fat.mp. \\
95 waist-height ratio*.mp. \\
96 waist to height ratio*.mp. \\
97 adipos*.mp. \\
98 body size*mp. \\
99 waist circumference*.mp. \\
100 hip circumference*.mp. \\
101 weight*.mp. \\
102 height*.mp. \\
103 waist-hip ratio*.mp. \\
104 waist to hip ratio*.mp. \\
105 skinfold thickness*.mp. \\
10689 or 90 or 91 or 92 or 93 or 94 or 95 or 96 or 97 or 98 or 99 or \\
100 or 101 or 102 or 103 or 104 or 105 \\
107 10 and 50 and 56 and 88 and 106 \\
\hline
\end{tabular}

\section{Search strategy}

We will consult a Health Sciences librarian with expertise in systematic reviews when designing the search strategy. A proposed search strategy for MEDLINE is described in Table 1. Searches will be conducted in PubMed, MEDLINE, EMBASE, PsycINFO, SPORTDiscus, CINAHL, Cochrane Database of Systematic Review, Cochrane Central Register of Controlled Trials (CENTRAL), and Database of Abstracts of Reviews of Effect (DARE). We will search ClinicalTrials.gov and ProQuest Dissertations and Theses A\&I to identify relevant gray literature. We will also search the reference lists of articles deemed eligible for inclusion in the analysis for relevant studies.

\section{Data management}

Two independent reviewers will perform data abstraction and quality assessment. Disagreement between the two reviewers will be resolved by discussion, with subsequent involvement of a third reviewer to arbitrate disagreements. Excel spreadsheets will be used to manage study records during the screening process. We will use the Grading of Recommendations Assessment, Development and Evaluation Profiler (GRADEpro) software to create tables for summary of findings and quality assessment [42].

\section{Data screening}

Duplicates will be removed, followed by screening of titles and abstracts. Full-text articles that meet the inclusion criteria will be retrieved and screened. Screening at all steps will be conducted independently by two reviewers, who will meet after each step to ensure consistency and to resolve conflicts. In the case of persisting disagreement, a third reviewer will be consulted. A flow diagram will be included to report the screening process (Fig. 1) [43, 44].

\section{Data abstraction}

Data will be extracted independently by two reviewers, using a data abstraction form specifically designed for this systematic review. Details to be collected include title, authors, publication date, journal name, setting, country, funding source, study design, study duration, eligibility criteria, sample size, and methods used for brain tumor diagnosis including imaging, histology, and clinical assessment.

Participants' characteristics include age at diagnosis of brain tumor and at study enrollment, sex, ethnicity, and brain tumor location and laterality. Treatment details include radiotherapy type (fractionated or non-fractionated) and dose, chemotherapy type, dose and duration, and surgery details (total resection, partial resection, shunting, ventriculostomy, others).

Detailed description of the obesity interventions will be recorded including study design, components, duration, and adverse events. We will document primary and secondary outcomes of the studies. Adjustment for confounders and details of the statistical analyses performed will be extracted as well as study results. We will attempt to retrieve incomplete data by contacting the corresponding authors of published work.

\section{Quality assessment}

The Risk of Bias Assessment Tool from the Cochrane Collaboration will be used to assess RCT [45]. This tool includes six domains: sequence generation, allocation concealment, blinding, incomplete data, selective reporting outcomes, and other sources of bias. Each RCT will be rated as having either a high, low, or unclear risk of bias.

The Risk of Bias In Non-randomized Studies-of Interventions (ROBINS-I) assessment tool will be used for non-randomized studies such as cohort studies [46]. This tool includes three domains: pre-intervention, atintervention, and post-intervention.

In the pre-intervention domain, bias due to confounding and participant selection are evaluated. Possible confounding factors include brain tumor location, type, treatments, years of survival, age, sex, pubertal stage, baseline body composition, and presence of comorbidities such as metabolic syndrome and hormonal 


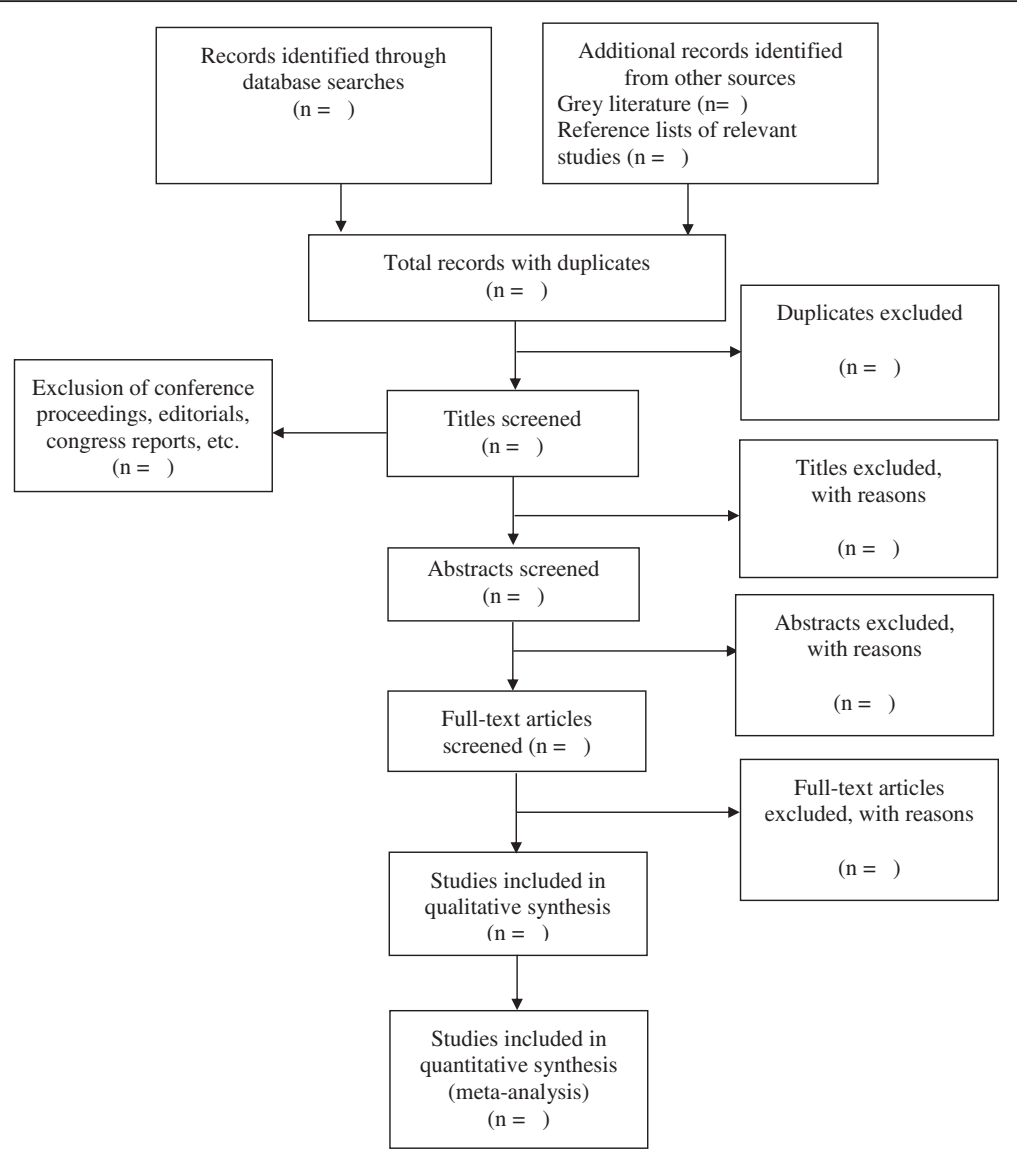

Fig. 1 Flow diagram of the article screening process

deficiency. Bias due to misclassification of the intervention status is assessed in the at-intervention domain. The post-intervention domain includes bias due to departures from the intended interventions, missing data, methods of outcome measurements, and selective reporting outcomes. In particular, co-interventions between lifestyle interventions, pharmacotherapy, and bariatric surgery can contribute to bias during the post-intervention domain. For example, participants may take antiobesity agents while they are on diet restriction. Each non-randomized study will be rated as having either a low, moderate, serious, critical, or unclear risk of bias.

The quality of uncontrolled studies will be assessed with a checklist developed by the University of Alberta Evidence-based Practice Center (UAEPC) [47]. This checklist evaluates selection bias, incomplete data, and the methods of outcome assessments. We will tabulate risk of bias for all included studies and discuss its impact on the meta-analysis.

The quality of evidence will be assessed using the Grading quality of evidence and strength of recommendations (GRADE) guidelines [48]. The GRADE guideline covers risk of bias, inconsistency, indirectness, imprecision, and publication bias. The overall quality of evidence is reported by each outcome measure as high, moderate, low, or very low.

\section{Data analysis}

Detailed characteristics of the included studies will be provided, in addition to a meta-analysis if applicable. We will analyze each intervention separately, and outcomes will be analyzed separately based on study designs. We will perform a meta-analysis if two or more studies are identified per intervention.

Dichotomous outcomes will be reported as odds ratio, while continuous outcomes will be reported as standardized mean differences and $95 \%$ confidence intervals. Expecting high levels of heterogeneity, our primary approach will emphasize the random effects estimate if more than ten studies can be identified [49]. Otherwise, both random effect and fixed effect models will be presented.

Inconsistency index $\left(I^{2}\right)$ and $P$ values will be used to quantify heterogeneity. The interpretation of the $I^{2}$ will be based on the threshold set by the Cochrane 
Collaboration [50]. If appropriate, a stratified analysis by sex will be pursued to identify a source of heterogeneity, as female SCBT are more at risk of developing obesity than males $[8,10]$.

If sufficient studies are identified for an outcome $(\geq 10)$, we will perform sensitivity analysis by excluding outlier, small-sized, or highly biased studies to determine the impact of these studies on the meta-analysis result. To investigate publication bias, we will create a contourenhanced funnel plot and use Egger's test and visual inspection to determine plot asymmetry, if there are ten or more studies for an outcome [51].

All meta-analyses will be conducted using Review Manager software version 5.3 (RevMan 5.3) [52] while Comprehensive Meta-Analysis software version 3 (CMA 3.0) will be used for Egger's test [53]. When metaanalysis is not appropriate, a table for summary of findings will be created using GRADEpro software and a narrative summary will be reported. The results of this systematic review will be presented according to the Preferred Reporting Items for Systematic Reviews and Meta-Analyses (PRISMA) guidelines [43, 44]. When amendments of the protocol are needed, we will document the date and the rationale for these changes.

\section{Discussion}

As the number of SCBT increased over time, it has become apparent that the burden of surviving a brain tumor is significant $[4,6,12,13]$. Obesity is a critical comorbidity to address in survivors, as it drives the risk of cardiovascular diseases, type 2 diabetes, metabolic syndrome, and hypertension [7, 8, 17-19]. This reduces the quality of life and lifespan of the survivors and increases healthcare system utilization.

In order to improve health outcomes in SCBT, it is important to develop evidence-based interventions to treat and prevent obesity and its cardiometabolic comorbidities.

The findings from this systematic review will have important implications for SCBT, as it will provide insights into the current best form of obesity intervention for these patients. The review will also define gaps in knowledge and help improve the quality of life and lifespan of SCBT by guiding the design of new interventions to target obesity and its cardiometabolic comorbidities.

\section{Additional file}

Additional file 1: PRISMA-P checklist. This checklist includes recommended items to address in a systematic reviews protocol and their location in this protocol. (DOCX $40 \mathrm{~kb})$

\section{Abbreviations}

BMI, body mass index; CENTRAL, Cochrane Central Register of Controlled Trials; CINAHL, Cumulative Index to Nursing and Allied Health Literature; CMA 3.0, Comprehensive Meta-Analysis version 3; DARE, Database of
Abstracts of Reviews of Effect; EMBASE, Excerpta Medica Database; GRADE, Grading of Recommendations Assessment, Development and Evaluation; PRISMA, Preferred Reporting Items for Systematic Review and Meta-Analyses; $\mathrm{RCT}$, randomized controlled trial; RevMan 5.3, Review Manager software version 5.3; ROBINS-I, Risk Of Bias in Non-randomized Studies_-of Interventions; SCBT, survivors of childhood brain tumors; UAEPC, University of Alberta Evidence-based Practice Center

\section{Acknowledgements}

We thank Dena Zeraatkar for the assistance with resources for quality and risk of bias assessments.

\section{Funding}

This protocol has received no funding.

\section{Availability of data and materials}

This is an Open Access article distributed under the terms of the Creative Commons Attribution License, which permits unrestricted use, distribution, and reproduction in any medium, provided the original work is properly credited. We will provide the data to interested parties upon request.

\section{Authors' contributions}

MCS is the guarantor. Research question was defined by MCS, AF, SKS, SB, and LT. Search strategy and eligibility criteria were developed by KWW, MV, RC, LB, and MCS. Data abstraction form was designed by KWW, MV, RC, and MCS. Quality and risk of bias assessments were conducted by KWW and RC. Methodological support was provided by RJdeS and LT. KWW, RC, AF, SKS $S B, L T, L B$, and MCS drafted the manuscript. All authors reviewed, edited, and approved the final version of the manuscript.

Competing interests

The authors declare that they have no competing interests.

\section{Author details}

${ }^{1}$ Department of Pediatrics, McMaster University, 1280 Main Street West, HSC-3A57, Hamilton, Ontario L8S 4K1, Canada. ${ }^{2}$ Division of Pediatric Endocrinology, McMaster Children's Hospital, 1280 Main Street West, HSC-3A57, Hamilton, Ontario L8S 4K1, Canada. ${ }^{3}$ Health Sciences Library, McMaster University, Hamilton, Ontario, Canada. ${ }^{4}$ Division of Pediatric Hematology/Oncology, McMaster Children's Hospital, Hamilton, Ontario, Canada. ${ }^{5}$ Division of Neurosurgery, Department of Surgery, McMaster Children's Hospital, Hamilton, Ontario, Canada. ${ }^{6}$ McMaster Stem Cell and Cancer Research Institute, McMaster University, Hamilton, Ontario, Canada. ${ }^{7}$ Division of Orthopedic Surgery, Department of Surgery, McMaster University Medical Centre, Hamilton, Ontario, Canada. ${ }^{8}$ Department of Clinical Epidemiology and Biostatistics, McMaster University, Hamilton, Ontario,

Canada. ${ }^{9}$ Department of Anesthesia, McMaster University, Hamilton, Ontario, Canada. ${ }^{10}$ Centre for Evaluation of Medicines, St. Joseph's Healthcare Hamilton, Hamilton, Ontario, Canada. ${ }^{11}$ Biostatistics Unit, St Joseph's Healthcare Hamilton, Hamilton, Ontario, Canada.

Received: 8 March 2016 Accepted: 27 May 2016

Published online: 14 June 2016

\section{References}

1. McKinney PA. Brain tumours: incidence, survival, and aetiology. J Neurol Neurosurg Psychiatry. 2004;75 Suppl 2:ii12-7.

2. Dolecek TA, Propp JM, Stroup NE, Kruchko C. CBTRUS statistical report: primary brain and central nervous system tumors diagnosed in the United States in 2005-2009. Neuro Oncol. 2012;14 Suppl 5:v1-49.

3. Woehrer A, Hackl M, Waldhor T, Weis S, Pichler J, Olschowski A, et al. Relative survival of patients with non-malignant central nervous system tumours: a descriptive study by the Austrian Brain Tumour Registry. $\mathrm{Br}$ J Cancer. 2014:110(2):286-96.

4. Mertens AC, Yasui Y, Neglia JP, Potter JD, Nesbit Jr ME, Ruccione K, et al. Late mortality experience in five-year survivors of childhood and adolescent cancer: the Childhood Cancer Survivor Study. J Clin Oncol. 2001;19(13): 3163-72.

5. Prasad PK, Signorello LB, Friedman DL, Boice Jr JD, Pukkala E. Long-term non-cancer mortality in pediatric and young adult cancer survivors in Finland. Pediatr Blood Cancer. 2012;58(3):421-7. 
6. Samaan MC, Akhtar-Danesh N. The impact of age and race on longevity in pediatric astrocytic tumors: a population-based study. Pediatr Blood Cancer. 2015;62(9):1567-71.

7. Bowers DC, Liu Y, Leisenring W, McNeil E, Stovall M, Gurney JG, et al. Lateoccurring stroke among long-term survivors of childhood leukemia and brain tumors: a report from the Childhood Cancer Survivor Study. J Clin Oncol. 2006;24(33):5277-82.

8. Pietilä S, Mäkipernaa A, Sievänen H, Koivisto AM, Wigren T, Lenko HL. Obesity and metabolic changes are common in young childhood brain tumor survivors. Pediatr Blood Cancer. 2009;52(7):853-9.

9. Oeffinger KC, Mertens AC, Sklar CA, Kawashima T, Hudson MM, Meadows AT, et al. Chronic health conditions in adult survivors of childhood cancer. N Engl J Med. 2006;355(15):1572-82.

10. Lek N, Prentice P, Williams RM, Ong KK, Burke GA, Acerini CL. Risk factors for obesity in childhood survivors of suprasellar brain tumours: a retrospective study. Acta Paediatr. 2010;99(10):1522-6.

11. Lustig RH, Post SR, Srivannaboon K, Rose SR, Danish RK, Burghen GA, et al. Risk factors for the development of obesity in children surviving brain tumors. J Clin Endocrinol Metab. 2003;88(2):611-6.

12. Ono N, Kohga H, Zama A, Inoue HK, Tamura M. A comparison of children with suprasellar germ cell tumors and craniopharyngiomas: final height, weight, endocrine, and visual sequelae after treatment. Surg Neurol. 1996; 46(4):370-7.

13. Sterkenburg AS, Hoffmann A, Gebhardt U, Warmuth-Metz M, Daubenbuchel AM, Muller HL. Survival, hypothalamic obesity, and neuropsychological/ psychosocial status after childhood-onset craniopharyngioma: newly reported long-term outcomes. Neuro Oncol. 2015;17(7):1029-38.

14. Ward BW, Schiller JS, Freeman G. Early release of selected estimates based on data from the January-September 2013 National Health Interview Survey: National Center for Health Statistics. 2014 [cited May 2016]. Available from: http://www.cdc.gov/nchs/data/nhis/earlyrelease/earlyrelease201403. pdf. Accessed 5 Jun 2016.

15. Wilson CL, Liu W, Yang JJ, Kang G, Ojha RP, Neale GA, et al. Genetic and clinical factors associated with obesity among adult survivors of childhood cancer: a report from the St. Jude Lifetime Cohort. Cancer. 2015;121(13): 2262-70.

16. An R. Health care expenses in relation to obesity and smoking among U.S. adults by gender, race/ethnicity, and age group: 1998-2011. Public Health 2015;129(1):29-36

17. Brown CD, Higgins M, Donato KA, Rohde FC, Garrison R, Obarzanek E, et al. Body mass index and the prevalence of hypertension and dyslipidemia. Obes Res. 2000;8(9):605-19.

18. Poirier P, Giles TD, Bray GA, Hong Y, Stern JS, Pi-Sunyer FX, et al. Obesity and cardiovascular disease: pathophysiology, evaluation, and effect of weight loss. Arterioscler Thromb Vasc Biol. 2006;26(5):968-76.

19. Chambless LB, Parker SL, Hassam-Malani L, McGirt MJ, Thompson RC. Type 2 diabetes mellitus and obesity are independent risk factors for poor outcome in patients with high-grade glioma. J Neurooncol. 2012;106(2): 383-9.

20. Armstrong TS, Ying Y, Wu J, Acquaye AA, Vera-Bolanos E, Gilbert MR, et al. The relationship between corticosteroids and symptoms in patients with primary brain tumors: utility of the Dexamethasone Symptom Questionnaire-Chronic. Neuro Oncol. 2015;17(8):1114-20.

21. Hansen JA, Stancel HH, Klesges LM, Tyc VL, Hinds PS, Wu S, et al. Eating behavior and BMI in adolescent survivors of brain tumor and acute lymphoblastic leukemia. J Pediatr Oncol Nurs. 2014;31(1):41-50.

22. Mertens AC, Yasui $Y$, Liu $Y$, Stovall M, Hutchinson R, Ginsberg J, et al. Pulmonary complications in survivors of childhood and adolescent cancer. A report from the Childhood Cancer Survivor Study. Cancer. 2002;95(11):2431-41.

23. Miller TL, Lipsitz SR, Lopez-Mitnik G, Hinkle AS, Constine LS, Adams MJ, et al. Characteristics and determinants of adiposity in pediatric cancer survivors. Cancer Epidemiol Biomarkers Prev. 2010; 19(8):2013-22.

24. Mulrooney DA, Yeazel MW, Kawashima T, Mertens AC, Mitby P, Stovall M, et al. Cardiac outcomes in a cohort of adult survivors of childhood and adolescent cancer: retrospective analysis of the Childhood Cancer Survivor Study cohort. BMJ. 2009;339:b4606.

25. Schmitz KH, Holtzman J, Courneya KS, Masse LC, Duval S, Kane R. Controlled physical activity trials in cancer survivors: a systematic review and metaanalysis. Cancer Epidemiol Biomarkers Prev. 2005;14(7):1588-95.
26. Oberfield SE, Sklar CA. Endocrine sequelae in survivors of childhood cancer. Adolesc Med. 2002;13(1):161-9. viii.

27. Mulrooney DA, Ness KK, Neglia JP, Whitton JA, Green DM, Zeltzer LK, et al. Fatigue and sleep disturbance in adult survivors of childhood cancer: a report from the childhood cancer survivor study (CCSS). Sleep. 2008; 31(2):271-81.

28. Geenen MM, Cardous-Ubbink MC, Kremer LC, van den Bos C, van der Pal $\mathrm{HJ}$, Heinen RC, et al. Medical assessment of adverse health outcomes in long-term survivors of childhood cancer. JAMA. 2007;297(24):2705-15.

29. Green DM, Cox CL, Zhu L, Krull KR, Srivastava DK, Stovall M, et al. Risk factors for obesity in adult survivors of childhood cancer: a report from the Childhood Cancer Survivor Study. J Clin Oncol. 2012;30(3):246-55.

30. Bray GA. Predicting obesity in adults from childhood and adolescent weight. Am J Clin Nutr. 2002;76(3):497-8.

31. Dietz WH, Robinson TN. Clinical practice. Overweight children and adolescents. N Engl J Med. 2005;352(20):2100-9.

32. Guo SS, Wu W, Chumlea WC, Roche AF. Predicting overweight and obesity in adulthood from body mass index values in childhood and adolescence. Am J Clin Nutr. 2002;76(3):653-8.

33. Nader PR, O'Brien M, Houts R, Bradley R, Belsky J, Crosnoe R, et al. Identifying risk for obesity in early childhood. Pediatrics. 2006;118(3): e594-601.

34. Simmonds M, Llewellyn A, Owen CG, Woolacott N. Predicting adult obesity from childhood obesity: a systematic review and meta-analysis. Obes Rev. 2016;17(2):95-107.

35. Shamseer L, Moher D, Clarke M, Ghersi D, Liberati A, Petticrew M, et al. Preferred reporting items for systematic review and meta-analysis protocols (PRISMA-P) 2015: elaboration and explanation. BMJ. 2015;349:97647.

36. Barlow SE, Expert C. Expert committee recommendations regarding the prevention, assessment, and treatment of child and adolescent overweight and obesity: summary report. Pediatrics. 2007;120 Suppl 4:S164-92.

37. Reeves BC, Deeks JJ, Higgins JPT, Wells GA. Chapter 13: Including nonrandomized studies. In: Higgins JPT, Green S, editors. Cochrane handbook for systematic reviews of interventions version 5.1.0. [updated March 2011]: The Cochrane Collaboration. 2011. Available from http://handbook. cochrane.org/. Accessed 5 Jun 2016.

38. Kang JG, Park CY. Anti-Obesity Drugs: A Review about Their Effects and Safety. Diabetes Metab J. 2012;36(1):13-25.

39. Hopkins JC, Howes N, Chalmers K, Savovic J, Whale K, Coulman KD, et al. Outcome reporting in bariatric surgery: an in-depth analysis to inform the development of a core outcome set, the BARIACT Study.Obesity Reviews. 2015;16(1):88-106.

40. Ried-Larsen M, Christensen R, Hansen KB, Johansen MY, Pedersen M, Zacho $\mathrm{M}$, et al. Head-to-head comparison of intensive lifestyle intervention ( $U$ TURN) versus conventional multifactorial care in patientswith type 2 diabetes: protocol and rationale for an assessor-blinded, parallel group and randomised trial. BMJ Open. 2015;5(12).

41. Yang Z, Scott CA, Mao C, Tang J, Farmer AJ. Resistance Exercise Versus Aerobic Exercise for Type 2 Diabetes: A Systematic Review and MetaAnalysis. Sports Medicine. 2014;44(4):487-99.

42. GRADEpro. Computer program on http://gradepro.org/. Version May 2016. McMaster University. 2014. Accessed 5 Jun 2016.

43. Liberati A, Altman DG, Tetzlaff J, Mulrow C, Gotzsche PC, loannidis JP, et al. The PRISMA statement for reporting systematic reviews and meta-analyses of studies that evaluate healthcare interventions: explanation and elaboration. BMJ. 2009;339:b2700.

44. Moher D, Liberati A, Tetzlaff J, Altman DG, Group P. Preferred reporting items for systematic reviews and meta-analyses: the PRISMA statement. Int J Surg. 2010;8(5):336-41.

45. Higgins JPT, Altman DG, Sterne JAC (editors). Chapter 8: Assessing risk of bias in included studies. In: Higgins JPT, Green S, editors. Cochrane handbook for systematic review of interventions Version 5.1.0. [updated March 2011]: The Cochrane Collaboration. 2011. Available from www. cochrane-handbook.org. Accessed 5 Jun 2016.

46. Sterne JAC, Higgins JPT, Reeves BC on behalf of the development group for ROBINS-l: a tool for assessing Risk Of Bias In Non-randomized Studies of Interventions, Version 7 March 2016 [cited May 2016]. Available from: hhttps://sites.google.com/site/riskofbiastool/. Accessed 5 Jun 2016.

47. Seida JC, Schouten JR, Mousavi SS, Tjosvold L, Vandermeer B, Milne A, et al. Comparative effectiveness of nonoperative and operative treatment for rotator cuff tears [Internet]. Rockville (MD): Agency for Healthcare Research 
and Quality (US); 2010. (Comparative Effectiveness Reviews, No. 22.) 2, Methods. Available from: http://www.ncbi.nlm.nih.gov/books/NBK47298/. Accessed 5 Jun 2016.

48. Atkins $D$, Best $D$, Briss PA, Eccles M, Falck-Ytter $Y$, Flottorp $S$, et al. Grading quality of evidence and strength of recommendations. BMJ. 2004;328(7454):1490

49. Villar J, Mackey ME, Carroli G, Donner A. Meta-analyses in systematic reviews of randomized controlled trials in perinatal medicine: comparison of fixed and random effects models. Stat Med. 2001;20(23):3635-47.

50. Deeks JJ, Higgins JPT, Altman DG (editors). Chapter 9: Analysing data and undertaking meta-analyses. In: Higgins JPT, Green S, editors. Cochrane handbook for systematic reviews of interventions version 5.1.0. [updated March 2011 ]: The Cochrane Collaboration, 2011. Available from www.cochrane-handbook.org. Accessed 5 Jun 2016.

51. Sterne JAC, Egger M, Moher D (editors). Chapter 10: Addressing reporting biases. In: Higgins JPT, Green S, editors. Cochrane handbook for systematic reviews of intervention version 5.1.0. [updated March 2011]: The Cochrane Collaboration, 2011. Available from www.cochrane-handbook.org. Accessed 5 Jun 2016.

52. Review Manager (RevMan) [Computer program]. Version 5.3. Copenhagen: The Nordic Cochrane Centre, The Cochrane Collaboration; 2014.

53. Comprehensive Meta-Analysis (CMA) [Computer program]. Version 3. Englewood NJ: Biostat.

\section{Submit your next manuscript to BioMed Central and we will help you at every step:}

- We accept pre-submission inquiries

- Our selector tool helps you to find the most relevant journal

- We provide round the clock customer support

- Convenient online submission

- Thorough peer review

- Inclusion in PubMed and all major indexing services

- Maximum visibility for your research

Submit your manuscript at www.biomedcentral.com/submit
Biomed Central 